TITLE:

\title{
Yangian symmetry in deformed WZNW models on squashed spheres
}

$\operatorname{AUTHOR}(S)$ :

Kawaguchi, lo; Orlando, Domenico; Yoshida, Kentaroh

\section{CITATION:}

Kawaguchi, lo ... [et al]. Yangian symmetry in deformed WZNW models on squashed spheres. Physics Letters B 2011, 701(4): 475-480

ISSUE DATE:

2011-07

URL:

http://hdl.handle.net/2433/143739

\section{RIGHT:}

(C) 2011 Elsevier B.V.; This is not the published version. Please cite only the published version.; この論文は出版社版でありません。引用の際に は出版社版をご確認ご利用ください。 
KUNS-2328

IPMU11-0054

\title{
Yangian symmetry in deformed WZNW models on squashed spheres
}

\author{
Io Kawaguchi $^{\dagger}$, Domenico Orlando* and Kentaroh Yoshida ${ }^{\dagger}$ \\ ${ }^{\dagger}$ Department of Physics, Kyoto University \\ Kyoto 606-8502, Japan \\ *Institute for the Physics and Mathematics of the Universe (IPMU), \\ The University of Tokyo, Kashiwa, Chiba 277-8568, Japan.
}

\begin{abstract}
We introduce a deformation of the Wess-Zumino-Novikov-Witten model with threedimensional squashed sphere target space. We show how with an appropriate choice of Wess-Zumino and boundary terms it is possible to construct an infinite family of conserved charges realizing an SU(2) Yangian. Finally we discuss the running of the squashing parameter under renormalization group flow.
\end{abstract}




\section{Introduction}

In 1978, Lüscher and Pohlmeyer found that the $\mathrm{O}(N)$ non-linear sigma model in two dimensions has a hidden infinite-dimensional symmetry [1] (for related work, see [2,3] and the comprehensive textbook [4]). This symmetry received a mathematical formulation in the work of Drinfel'd [5], who called it Yangian. A similar construction can be extended to non-linear sigma models on symmetric spaces where it is again possible to construct an infinite number of non-local charges which realize a Yangian. An important point to note here is that a flat conserved current exists always from which an infinite number of non-local charges can be generated, for example by following the treatment in [6]. Since AdS spaces and round spheres are symmetric spaces, the same argument can be used in the AdS/CFT correspondence [7] as pointed out in [8,9] and extensively discussed in [10], where a list of symmetric spaces with potential AdS/CFT application is given.

While the symmetric cases have been studied intensively and are well understood, finding hidden infinite-dimensional symmetries for non-symmetric cosets is still an open problem. These are of physical interest and have been intensively studied in recent years. Typical examples are the Schrödinger and Lifshitz spacetimes [11-14] that appear in applications of the AdS/CFT correspondence to condensed matter physics. Other examples of non-symmetric cosets are three-dimensional squashed spheres and warped AdS spaces. In topologically massive gravity [15] for example, warped AdS spaces appear as classical solutions [16]. They also appear as the near-horizon geometry of the extremal Kerr black hole $[17,18]$, and play an important role in the Kerr/CFT correspondence [18]. In addition, squashed spheres and warped AdS spaces appear in string theory contexts such as deformations of heterotic string backgrounds [19-24], T-dualities of some string backgrounds [25], as well as in relation to condensed matter system [26].

Recently, it has been shown that the Yangian symmetry is preserved in a non-linear sigma model on a three-dimensional squashed sphere [27]:

$$
\mathrm{d} s^{2}=\frac{L^{2}}{4}\left[\mathrm{~d} \theta^{2}+\cos ^{2} \theta \mathrm{d} \phi^{2}+(1+C)(\mathrm{d} \psi+\sin \theta \mathrm{d} \phi)^{2}\right] .
$$

This geometry is a deformation of a three-dimensional round sphere $S^{3}$, where the constant parameter $C$ measures how much the original round sphere is squashed. The round sphere is realized for $C=0$ and its isometry is $\mathrm{SO}(4)=\mathrm{SU}(2)_{\mathrm{L}} \times \mathrm{SU}(2)_{\mathrm{R}}$. For $C \neq 0$, the isometry is reduced to $\mathrm{SU}(2)_{\mathrm{L}} \times \mathrm{U}(1)_{\mathrm{R}}$. Recall that the presence of a flat conserved current is crucial for the realization of the Yangian symmetry. In the case discussed in [27] it has been shown that the flat conserved current can be constructed by improving the Noether current only for $C \geq 0$. On the other hand, the positivity of $C$ might seem strange since the preferred range of $C$ is $-1 \leq C \leq 0$ in physical setups such as the deformation of the heterotic string background discussed in [19]. Also in the recent T-duality argument on how to realize a Yangian symmetry with a squashed sphere target space [28], the range of $C$ is naturally restricted to $-1 \leq C \leq 0$. It is therefore an interesting question to consider how the Yangian symmetry can be realized in this range. 
Here we argue that a Wess-Zumino (wz) term should be added to the sigma model action. The theory with the wz term can be called the squashed Wess-Zumino-NovikovWitten (WZNW) model. Our main result is that for an appropriate choice of the normalization of the wz term (which is constrained by dimensionality ad quantum consistency) and adding a boundary term it is in general possible to construct a flat conserved current. This is equivalent to saying that the Yangian algebra can be realized even for $-1 \leq C \leq 0$.

This letter is organized as follows. In Section 2 we introduce the action of the wZNW model on a three-dimensional squashed sphere. Then we show that a flat conserved current can be constructed i) for a certain value of the coefficient of the wz term and ii) for general values by improving the Noether current. In Section 3 the Yangian algebra is computed with the standard Poisson bracket. In Section 4 the renormalization group flow is discussed by computing one-loop $\beta$-functions. Section 5 is devoted to conclusion and discussion.

\section{The flatness condition for the squashed wznw model}

Consider the action of a two-dimensional sigma model with squashed sphere target space:

$$
S_{\sigma \mathrm{M}}=-\frac{1}{2 \lambda^{2}} \iint \mathrm{d} t \mathrm{~d} x\left[\left(\partial_{\mu} \theta\right)^{2}+\cos ^{2} \theta\left(\partial_{\mu} \phi\right)^{2}+(1+C)\left(\partial_{\mu} \psi+\sin \theta \partial_{\mu} \phi\right)^{2}\right] .
$$

The base space is a two-dimensional Minkowski spacetime with coordinates $x^{\mu}=(t, x)$ and metric $\eta_{\mu v}=(-1,+1)$. The parameter $\lambda^{2}$ is the bare coupling constant.

It is convenient to introduce the $\mathrm{SU}(2)_{\mathrm{L}}$ group element

$$
g=\mathrm{e}^{\phi T_{1}} \mathrm{e}^{\theta T_{2}} \mathrm{e}^{\psi T_{3}}, \quad g \in \mathrm{SU}(2)_{\mathrm{L}},
$$

where the $\mathrm{SU}(2)_{\mathrm{L}}$ generators $T_{A}(A=1,2,3)$ satisfy the relations

$$
\left[T_{A}, T_{B}\right]=\epsilon_{A B}{ }^{C} T_{C}, \quad \operatorname{Tr}\left(T_{A} T_{B}\right)=-\frac{1}{2} \delta_{A B},
$$

where $\epsilon_{A B}{ }^{C}$ is the anti-symmetric tensor. By using the left-invariant current $J_{\mu}$ on $\mathrm{SU}(2)_{\mathrm{L}}$ given by

$$
J_{\mu}=g^{-1} \partial_{\mu} g,
$$

the sigma model action is rewritten as

$$
S_{\sigma \mathrm{M}}=\frac{1}{\lambda^{2}} \iint \mathrm{d} t \mathrm{~d} x \eta^{\mu \nu}\left[\operatorname{Tr}\left(J_{\mu} J_{v}\right)-2 C \operatorname{Tr}\left(T_{3} J_{\mu}\right) \operatorname{Tr}\left(T_{3} J_{v}\right)\right]
$$

Next let us introduce the Wess-Zumino (wz) term,

$$
S_{\mathrm{wZ}} \equiv \frac{n}{12 \pi} \int_{0}^{1} \mathrm{~d} s \iint \mathrm{d} t \mathrm{~d} x \epsilon_{\hat{\mu} \hat{v} \hat{\rho}} \operatorname{Tr}\left(J_{s}^{\hat{\mu}} J_{s}^{\hat{v}} J_{s}^{\hat{\rho}}\right) \quad(n \in \mathbb{Z}),
$$

where $n$ is an integer. It is given by a three-form defined on a fictitious three-dimensional base space with coordinates $x^{\hat{\mu}}=\left(x^{\mu}, s\right)$. The anti-symmetric tensor $\epsilon_{\hat{\mu} \hat{v} \hat{\rho}}$ is normalized as 
$\epsilon_{t x s}=+1$. The coordinate $s$ describes an interval, $s \in[0,1]$. The variable $g\left(x^{\mu}, s\right) \equiv g_{s}\left(x^{\mu}\right)$ interpolates between the unit element and $g(x)$, i.e. $g_{0}(x)=1$ and $g_{1}(x)=g(x)$. The current $J_{s}^{\hat{\mu}}$ is defined as $J_{s}^{\hat{\mu}} \equiv g_{s}^{-1} \partial^{\hat{\mu}} g_{s}$.

Note that the wz term is the same as in the usual wzNw model because of dimensionality and quantum consistency. In terms of the angle variables, the integrand of the wz term is proportional to the volume form,

$$
\epsilon_{\hat{\mu} \hat{v} \hat{\rho}} \operatorname{Tr}\left(J_{s}^{\hat{\mu}} J_{s}^{\hat{v}} J_{s}^{\hat{\rho}}\right) \mathrm{d} t \mathrm{~d} x \mathrm{~d} s \propto \epsilon_{\hat{\mu} \hat{v} \hat{\rho}} \cos \theta_{s} \partial^{\hat{\mu}} \phi_{s} \partial^{\hat{v}} \theta_{s} \partial^{\hat{\rho}} \psi_{s} \mathrm{~d} t \mathrm{~d} x \mathrm{~d} s
$$

This is simply due to dimensionality. The target space is now three-dimensional and so the three-form to define the wz term must be proportional to the volume form. Thus the form of the wz term is essentially fixed by the dimensionality in the present case, up to an overall constant. This constant has to be discretized in the standard way. At the end of the day, the wZ term turns out to be exactly the same as in the SU(2) wZNw model.

The model described by the sum of $S_{\sigma \mathrm{M}}$ and $S_{\mathrm{wz}}$ can be called the squashed WessZumino-Novikov-Witten (WZNW) model. The resulting action is given by

$$
\begin{aligned}
& S_{\mathrm{Sq}}=S_{\sigma \mathrm{M}}+S_{\mathrm{wz}}, \\
& S_{\sigma \mathrm{M}}=\frac{1}{\lambda^{2}} \iint \mathrm{d} t \mathrm{~d} x \eta^{\mu v}\left[\operatorname{Tr}\left(J_{\mu} J_{v}\right)-2 C \operatorname{Tr}\left(T_{3} J_{\mu}\right) \operatorname{Tr}\left(T_{3} J_{v}\right)\right], \\
& S_{\mathrm{wz}}=\frac{n}{12 \pi} \int_{0}^{1} d s \iint \mathrm{d} t \mathrm{~d} x \epsilon_{\hat{\mu} \hat{v} \hat{\rho}} \operatorname{Tr}\left(J_{s}^{\hat{\mu}} J_{s}^{\hat{v}} J_{s}^{\hat{\rho}}\right), \quad(n \in \mathbb{Z}) .
\end{aligned}
$$

The corresponding equations of motion have the form

$$
\partial_{\mu} J^{\mu}-2 C \operatorname{Tr}\left(T_{3} \partial_{\mu} J^{\mu}\right) T_{3}+2 C \operatorname{Tr}\left(T_{3} J^{\mu}\right)\left[T_{3}, J_{\mu}\right]-\frac{K}{2} \epsilon^{\mu v}\left[J_{\mu}, J_{v}\right]=0,
$$

where $J^{\mu}=\eta^{\mu v} J_{v}$ and

$$
K \equiv \frac{n \lambda^{2}}{8 \pi}
$$

Note that $C=0, K=1$ is the fixed point for the standard wZNW model.

For $C \neq 0$, the Lagrangian has $\mathrm{SU}(2)_{\mathrm{L}} \times \mathrm{U}(1)_{\mathrm{R}}$ symmetry. For the $\mathrm{SU}(2)_{\mathrm{L}}$ part, the Noether current $j_{\mu}$ is given by

$$
j_{\mu}=\partial_{\mu} g \cdot g^{-1}-2 C \operatorname{Tr}\left(T_{3} J_{\mu}\right) g T_{3} g^{-1}-K \epsilon_{\mu \nu} \partial^{v} g \cdot g^{-1} .
$$

In terms of the current, the equations of motion take the form

$$
g^{-1} \partial_{\mu} j^{\mu} g=0
$$

or, equivalently

$$
\mathrm{d} * j=0 .
$$


This shows that as usual, the Noether current is defined up to the Hodge dual of an exact 1 -form. Hence we can add an improvement term $I_{\mu}$ :

$$
j_{\mu} \mapsto j_{\mu}+I_{\mu} ; \quad I_{\mu} \equiv A \epsilon_{\mu \nu} \partial^{\nu} f,
$$

where $A$ is an arbitrary constant and $f$ a scalar function. As pointed out in [27], the function $f$ must have the form

$$
f=g T_{3} g^{-1}
$$

in order to satisfy the flatness condition. This improvement term corresponds to a boundary term of the form

$$
S_{\text {BDRY }}=\frac{A}{\lambda^{2}} \int \mathrm{d} t \mathrm{~d} x \epsilon_{\mu \nu} \partial^{\mu} J_{3}^{\nu}
$$

The resulting improved current $\tilde{j}_{\mu}$ depends on the three parameters $C, K$ and $A$ and is given by

$$
\tilde{j}_{\mu}=j_{\mu}+I_{\mu}=\partial_{\mu} g \cdot g^{-1}-2 C \operatorname{Tr}\left(T_{3} J_{\mu}\right) g T_{3} g^{-1}-K \epsilon_{\mu \nu} \partial^{v} g \cdot g^{-1}+A \epsilon_{\mu \nu} \partial^{\nu}\left(g T_{3} g^{-1}\right) \text {. }
$$

In order to define an infinite family of conserved charges we require the improved current $\tilde{j}$ to be flat. Because of the SU(2) symmetry, the flatness condition reduces to a single condition on the three parameters:

$$
\epsilon^{\mu v}\left(\partial_{\mu} \tilde{j}_{v}-\tilde{j}_{\mu} \tilde{j}_{v}\right)=\left(C-\frac{C K^{2}}{1+C}-A^{2}\right) \epsilon_{\mu v} \operatorname{Tr}\left(T_{3}\left[J^{\mu}, J^{v}\right]\right) g T_{3} g^{-1}=0 .
$$

It is interesting to consider separately the two cases $A=0$ and $A \neq 0$, with and without the improvement term:

1. For $A=0$, supposing that $C \geq-1$, we obtain the condition

$$
C=K^{2}-1 \text {. }
$$

Thus, as opposed to the case without a wz term [27], a flat conserved current can be constructed even for negative values of $C$. This condition is reminiscent of the one discussed in [19], but it is not exactly the same since the model at hand is not conformal.

2. For $A \neq 0$, it is possible to construct a flat conserved current if $A$ is taken to be

$$
A^{2}=C\left(1-\frac{K^{2}}{1+C}\right) \text {. }
$$

In this expression we have to restrict the range of $C$ to $C>-1$. Since $K$ is nonvanishing, a negative value of $C$ is possible. The equation is of second order in $C$ and can be solved algebraically. The solutions are given by

$$
C_{A}^{ \pm}(\lambda)=\frac{1}{2}\left[A^{2}-1+K^{2} \pm \sqrt{4 A^{2}+\left(A^{2}-1+K^{2}\right)^{2}}\right]
$$

In the following we will keep the three constant parameters $C, K$ and $A$, but assume either of the above conditions to be satisfied. When referring to the current, we will always mean the improved current and omit the tilde for simplicity. 


\section{The Yangian algebra}

So far we have constructed a flat conserved current for $\mathrm{SU}(2)_{\mathrm{L}}$ by imposing appropriate conditions on the parameters. Given this, it is always possible to construct an infinite number of non-local conserved charges by following the prescription in [6]. For example, the Noether charge $Q_{(0)}^{A}$ and the first non-local charge $Q_{(1)}^{A}$ are given by

$$
\begin{aligned}
& Q_{(0)}^{A} \equiv \int \mathrm{d} x j_{t}^{A}(x), \\
& Q_{(1)}^{A} \equiv-\int \mathrm{d} x j_{x}^{A}(x)+\frac{1}{4} \iint \mathrm{d} x \mathrm{~d} y \epsilon(x-y) \epsilon_{B C}{ }^{A} j_{t}^{B}(x) j_{t}^{C}(y),
\end{aligned}
$$

where

$$
\epsilon(x-y)= \begin{cases}+1 & \text { if } x>y \\ -1 & \text { if } x<y\end{cases}
$$

The conservation law for $Q_{(1)}^{A}$ is a consequence of the flatness condition.

The Poisson brackets of the charges can be found via the current algebra. Imposing the flatness condition in Eq. (2.22), the currents form an algebra in terms of the standard Poisson bracket for the dynamical variables. It reads:

$$
\begin{aligned}
\left\{j_{t}^{A}(x), j_{t}^{B}(y)\right\}_{\mathrm{P}}= & \epsilon^{A B}{ }_{C} j_{t}^{C}(x) \delta(x-y)-2 K \delta^{A B} \partial_{x} \delta(x-y) \\
\left\{j_{t}^{A}(x), j_{x}^{B}(y)\right\}_{\mathrm{P}}= & \epsilon^{A B}{ }_{C} j_{x}^{C}(x) \delta(x-y)+\left(1+C+\frac{K^{2}}{1+C}\right) \delta^{A B} \partial_{x} \delta(x-y), \\
\left\{j_{x}^{A}(x), j_{x}^{B}(y)\right\}_{\mathrm{P}}= & -\left(C+\frac{K^{2}}{1+C}\right) \epsilon^{A B}{ }_{C} j_{t}^{C}(x) \delta(x-y)-2 K \epsilon^{A B}{ }_{C} j_{x}^{C}(x) \delta(x-y) \\
& -2 K \delta^{A B} \partial_{x} \delta(x-y) .
\end{aligned}
$$

Note that the result of [27] is reproduced for $K=0$ (no wz term). For $C=0$ (no squashing) and $K=1$, the right-moving current $j_{\mathrm{R}} \equiv\left(j_{t}+j_{x}\right) / 2$ vanishes (i.e. $\left.j_{t}=-j_{x}\right)$ and only the algebra for the left-moving current $j_{\mathrm{L}} \equiv\left(j_{t}-j_{x}\right) / 2=j_{t}$ remains.

The current algebra (3.4) leads to the following (Yangian) algebra for the charges:

$$
\begin{aligned}
& \left\{Q_{(0)}^{A}, Q_{(0)}^{B}\right\}_{P}=\epsilon^{A B}{ }_{C} Q_{(0)}^{C}, \\
& \left\{Q_{(0)}^{A}, Q_{(1)}^{B}\right\}_{P}=\epsilon^{A B}{ }_{C} Q_{(1)}^{C}, \\
& \left\{Q_{(1)}^{A}, Q_{(1)}^{B}\right\}_{P}=\epsilon^{A B}{ }_{C}\left[Q_{(2)}^{C}+\frac{1}{12} Q_{(0)}^{C}\left(Q_{(0)}\right)^{2}+2 K Q_{(1)}^{C}-\left(C+\frac{K^{2}}{1+C}\right) Q_{(0)}^{C}\right],
\end{aligned}
$$

where $Q_{(2)}^{A}$ is the second non-local charge defined as

$$
\begin{aligned}
& Q_{(2)}^{A} \equiv \frac{1}{12} \iiint \mathrm{d} x \mathrm{~d} y \mathrm{~d} z \epsilon(x-y) \epsilon(y-z) \delta_{B C} {\left[j_{t}^{A}(x) j_{t}^{B}(y) j_{t}^{C}(z)-j_{t}^{B}(x) j_{t}^{A}(y) j_{t}^{C}(z)\right] } \\
&+\frac{1}{2} \iint \mathrm{d} x \mathrm{~d} y \epsilon(x-y) \epsilon_{B C}{ }^{A} j_{t}^{B}(x) j_{x}^{C}(y) .
\end{aligned}
$$


It is straightforward to check that the $\mathrm{SU}(2)_{\mathrm{L}}$ Serre relations are satisfied:

$$
\begin{aligned}
\left\{\left\{Q_{(1)}^{+}, Q_{(1)}^{-}\right\}_{\mathrm{P}}, Q_{(1)}^{3}\right\}_{\mathrm{P}} & =\frac{1}{4} Q_{(0)}^{3}\left(Q_{(1)}^{+} Q_{(0)}^{-}-Q_{(1)}^{-} Q_{(0)}^{+}\right), \\
\left\{\left\{Q_{(1)}^{3}, Q_{(1)}^{ \pm}\right\}_{\mathrm{P}}, Q_{(1)}^{ \pm}\right\}_{\mathrm{P}} & =\frac{1}{4} Q_{(0)}^{ \pm}\left(Q_{(1)}^{3} Q_{(0)}^{ \pm}-Q_{(1)}^{ \pm} Q_{(0)}^{3}\right), \\
\left\{\left\{Q_{(1)}^{+}, Q_{(1)}^{-}\right\}_{\mathrm{P}}, Q_{(1)}^{ \pm}\right\}_{\mathrm{P}} & \pm 2\left\{\left\{Q_{(1)}^{3}, Q_{(1)}^{ \pm}\right\}_{\mathrm{P}}, Q_{(1)}^{3}\right\}_{\mathrm{P}} \\
& =\frac{1}{4} Q_{(0)}^{ \pm}\left(Q_{(1)}^{+} Q_{(0)}^{-}-Q_{(1)}^{-} Q_{(0)}^{+}\right) \pm \frac{1}{2} Q_{(0)}^{3}\left(Q_{(1)}^{3} Q_{(0)}^{ \pm}-Q_{(1)}^{ \pm} Q_{(0)}^{3}\right),
\end{aligned}
$$

where

$$
j_{\mu}^{ \pm} \equiv j_{\mu}^{1} \pm i j_{\mu}^{2}
$$

Thus in the squashed wZNw model, the $\mathrm{SU}(2)_{\mathrm{L}}$ Yangian symmetry is realized as an infinitedimensional symmetry.

In fact it is possible to rewrite the Yangian algebra in a standard form by using the fact that higher charges are defined up to $Q_{(0)}$ and shifting the first and second charges as follows:

$$
\begin{aligned}
& \widetilde{Q}_{(1)}=Q_{(1)}-K Q_{(0)}, \\
& \widetilde{Q}_{(2)}=Q_{(2)}-A^{2} Q_{(0)} .
\end{aligned}
$$

\section{The $\beta$-function of the squashed wznw model}

The model described by the action $S_{\mathrm{Sq}}$ in Eq. (2.8) is in general not conformal even though, as we have shown in the previous section, it preserves a Yangian symmetry. In this section we study the running of the squashing parameter $C$ and the coupling constant $\lambda$ and discuss the relation between the condition obtained in the previous section and the renormalization group (RG) flow.

As it is already the case for the standard wZNw model, the qualitative behavior can be understood semiclassically at one-loop level*. Consider the action in Eq. (2.9). First decompose the $\mathrm{SU}(2)$ group element $g$ into a classical solution $g_{0}$ and a quantum fluctuation $\xi$ as follows:

$$
g=g_{0} \mathrm{e}^{\lambda \xi} \quad \xi \in \operatorname{Lie}[\mathrm{SU}(2)]
$$

then expand the left-invariant current $J$ with respect to $\lambda$,

$$
\begin{aligned}
J & =g^{-1} \mathrm{~d} g \\
& =\mathrm{e}^{-\lambda \xi}\left(g_{0}^{-1} \mathrm{~d} g_{0}\right) \mathrm{e}^{\lambda \xi}+\lambda \int_{0}^{1} \mathrm{~d} t \mathrm{e}^{-t \lambda \xi}(\mathrm{d} \xi) \mathrm{e}^{t \lambda \xi} \\
& =J_{0}+\lambda\left(\mathrm{d} \xi+\left[J_{0}, \xi\right]\right)+\frac{\lambda^{2}}{2}\left(-[\xi, \mathrm{d} \xi]+\left[\left[J_{0}, \xi\right], \xi\right]\right)+\mathcal{O}\left(\lambda^{3}\right),
\end{aligned}
$$

${ }^{*}$ The RG flow is the Bianchi Ix Ricci flow in the axisymmetric case with a wz term. As such it was already analyzed in [32, 33]. 
where $J_{0}=g_{0}^{-1} \mathrm{~d} g_{0}$. By rewriting the fluctuation $\xi$ as

$$
\xi=\xi^{1} T_{1}+\xi^{2} T_{2}+\frac{1}{\sqrt{1+C}} \xi^{3} T_{3}
$$

and introducing the new vector notation $\vec{\xi}=\left(\xi^{1}, \xi^{2}, \xi^{3}\right)$, the action at second order in the fluctuation is given by

$$
S_{\text {quad }}=\iint \mathrm{d} t \mathrm{~d} x\left[-\frac{1}{2} \eta^{\mu v} \partial_{\mu} \vec{\xi} \cdot \partial_{\nu} \vec{\xi}+\vec{J}_{0 \mu} \cdot \vec{v}^{\mu}+\frac{C}{2} \vec{J}_{0 \mu} \mathrm{M}^{\mu v} \vec{J}_{0 v}\right]
$$

where the following quantities have been introduced:

$$
\begin{aligned}
\vec{J}_{0 \mu}= & \left(\begin{array}{c}
J_{0 \mu}^{1} \\
J_{0 \mu}^{2} \\
J_{0 \mu}^{3}
\end{array}\right), \\
\vec{v}_{\mu}= & \left(\begin{array}{c}
-\frac{1}{2}\left(\sqrt{1+C} \eta_{\mu \nu}-\frac{\lambda^{2} n}{8 \pi \sqrt{1+C}} \epsilon_{\mu v}\right)\left(\xi^{2} \partial^{v} \xi^{3}-\xi^{3} \partial^{v} \xi^{2}\right) \\
\frac{1}{2}\left(\sqrt{1+C} \eta_{\mu \nu}-\frac{\lambda^{2} n}{8 \pi \sqrt{1+C}} \epsilon_{\mu \nu}\right)\left(\xi^{1} \partial^{v} \xi^{3}-\xi^{3} \partial^{v} \xi^{1}\right) \\
-\frac{1}{2}\left((1-C) \eta_{\mu \nu}-\frac{\lambda^{2} n}{8 \pi} \epsilon_{\mu \nu}\right)\left(\xi^{1} \partial^{v} \xi^{2}-\xi^{2} \partial^{v} \xi^{1}\right)
\end{array}\right), \\
\mathrm{M}_{\mu v}= & \eta_{\mu v}\left(\begin{array}{ccc}
-\xi^{2} \xi^{2} & \xi^{1} \xi^{2} & -\frac{1}{2} \sqrt{1+C} \xi^{1} \xi^{3} \\
-\frac{1}{2} \sqrt{1+C} \xi^{2} & -\frac{1}{2} \xi^{1} \xi^{3}-\frac{1}{1+C} \xi^{2} \xi^{3} & -\frac{1}{2} \sqrt{1+C} \xi^{1} \xi^{1}+\xi^{2} \xi^{2}
\end{array}\right)+ \\
+ & \frac{\lambda^{2} n \epsilon_{\mu \nu}}{16 \pi \sqrt{1+C}}\left(\begin{array}{ccc}
0 & 0 & \xi^{1} \xi^{3} \\
0 & 0 & \xi^{2} \xi^{3} \\
\xi^{1} \xi^{3} & \xi^{2} \xi^{3} & 0
\end{array}\right) .
\end{aligned}
$$

The divergent part in the one-loop effective action can be evaluated as follows:

$$
\begin{aligned}
\mathrm{e}^{i W\left[g_{0}\right]}= & \mathrm{e}^{i S_{\mathrm{Sq}_{q}}\left[g_{0}\right]} \int[\mathrm{d} \xi] \mathrm{e}^{i S_{\text {quad }}\left[g_{0} ; \xi\right]} \\
= & \mathrm{e}^{i S_{\mathrm{Sq}}\left[g_{0}\right]} \int[\mathrm{d} \xi] \exp \left\{-\frac{i}{2} \int \mathrm{d}^{2} x \eta^{\mu v} \partial_{\mu} \vec{\xi} \cdot \partial_{\nu} \vec{\xi}\right\} \\
& \times\left[1+i \int \mathrm{d}^{2} x \vec{J}_{0 \mu} \cdot \vec{v}^{\mu}(x)-\frac{1}{2} \int \mathrm{d}^{2} x \int \mathrm{d}^{2} y \vec{J}_{0 \mu} \cdot \vec{v}^{\mu}(x) \vec{J}_{0 v} \cdot \vec{v}^{v}(y)\right. \\
& \left.+i \frac{C}{2} \int \mathrm{d}^{2} x \vec{J}_{0 \mu} \mathrm{M}^{\mu v} \vec{J}_{0 v}(x)+\mathcal{O}\left(\xi^{3}\right)\right] \\
= & \mathrm{e}^{i S_{\mathrm{sq}}\left[g_{0}\right]} \\
& \times\left\{1+\frac{i}{16 \pi}\left[1-C-\frac{1}{1+C}\left(\frac{\lambda^{2} n}{8 \pi}\right)^{2}\right] \log \frac{\Lambda^{2}}{\mu^{2}} \int \mathrm{d}^{2} x \eta^{\mu v}\left[J_{0 \mu}^{1} J_{0 v}^{1}+J_{0 \mu}^{2} J_{0 v}^{2}\right]\right. \\
& \left.+\frac{i}{16 \pi}\left[(1+C)^{2}-\left(\frac{\lambda^{2} n}{8 \pi}\right)^{2}\right] \log \frac{\Lambda^{2}}{\mu^{2}} \int \mathrm{d}^{2} x \eta^{\mu v} J_{0 \mu}^{3} J_{0 v}^{3}+\text { finite }\right\},
\end{aligned}
$$


where we have used the two-point function:

$$
\left\langle\xi^{A}(x) \xi^{B}(y)\right\rangle=-\int \frac{\mathrm{d}^{2} k}{(2 \pi)^{2}} \mathrm{e}^{-i k \cdot(x-y)} \frac{1}{k^{2}} .
$$

From the explicit expression we can read off the one-loop effective coupling constant and the squashing parameter:

$$
\begin{aligned}
& \lambda_{\mathrm{R}}^{2}=\lambda^{2}+\frac{\lambda^{4}}{8 \pi}\left(1-C-\frac{1}{1+C}\left(\frac{\lambda^{2} n}{8 \pi}\right)^{2}\right) \log \frac{\Lambda^{2}}{\mu^{2}}, \\
& C_{R}=C-\frac{\lambda^{2}}{4 \pi} C(1+C) \log \frac{\Lambda^{2}}{\mu^{2}},
\end{aligned}
$$

where $\Lambda$ and $\mu$ are the ultraviolet (UV) and the infrared (IR) cut-off, respectively. By deriving with respect to $\mu$ we obtain the one-loop $\beta$-functions for $\lambda_{\mathrm{R}}^{2}$ and $C_{\mathrm{R}}$ :

$$
\begin{aligned}
& \mu \frac{\partial \lambda_{\mathrm{R}}^{2}}{\partial \mu}=-\frac{\lambda_{\mathrm{R}}^{4}}{4 \pi}\left\{1-C_{\mathrm{R}}-\frac{1}{1+C_{\mathrm{R}}}\left(\frac{\lambda_{\mathrm{R}}^{2} n}{8 \pi}\right)^{2}\right\}, \\
& \mu \frac{\partial C_{\mathrm{R}}}{\partial \mu}=\frac{\lambda_{\mathrm{R}}^{2}}{2 \pi} C_{\mathrm{R}}\left(1+C_{\mathrm{R}}\right) .
\end{aligned}
$$

Note that the coefficient of the $\mathrm{wz}$ term is quantized since $\mathrm{SU}(2)$ is a compact group.

Before discussing the RG flow described by (4.12) and (4.13), let us recall the condition we obtained from the flatness of the $\mathrm{SU}(2)_{\mathrm{L}}$ Noether current. By writing $K$ explicitly in terms of the coupling constant $\lambda$, the flatness condition for the conserved current in Eq. (2.22) can be rewritten as

$$
C-\frac{C}{1+C}\left(\frac{n \lambda^{2}}{8 \pi}\right)^{2}-A^{2}=0
$$

and $C$ can be expressed as a function of $\lambda$ for fixed values of $A$ as in Eq. (2.23):

$$
C_{A}^{ \pm}(\lambda)=\frac{1}{2}\left[\left(A^{2}-1\right)+\left(\frac{n \lambda^{2}}{8 \pi}\right)^{2}\right] \pm \sqrt{A^{2}+\frac{1}{4}\left(A^{2}-1+\left(\frac{n \lambda^{2}}{8 \pi}\right)^{2}\right)^{2}}
$$

It is interesting to discuss the RG flow with respect to the flatness condition (4.14). A typical example $(n=20)$ is represented in Fig. 4 . There is a unique IR fixed point on the line $C=0$ for $\lambda_{R}=8 \pi / n$, which is the same as in the usual SU(2) wzNW model. As $n$ increases, the fixed point approaches $\lambda^{2}=0$ on the line $C=0$. It is worth noting that the critical surface is defined by $C>-1$ and the universality class is characterized by the unique IR fixed point. In other words, the flow remains in the same universality class as the $\mathrm{SU}(2)$ wzw model. The locus $C=-1$ deserves some special attention. As already shown in [19], it corresponds to a decompactification limit in which the squashed three-sphere degenerates to the direct product $S^{2} \times S^{1}$. In this case, the action $S_{\sigma \mathrm{M}}$ in Eq. (2.5) describes the well-studied non-linear sigma model on the coset $\mathrm{SU}(2) / \mathrm{U}(1) \sim S^{2}$, also known as the $\mathrm{O}(3)$ model. 


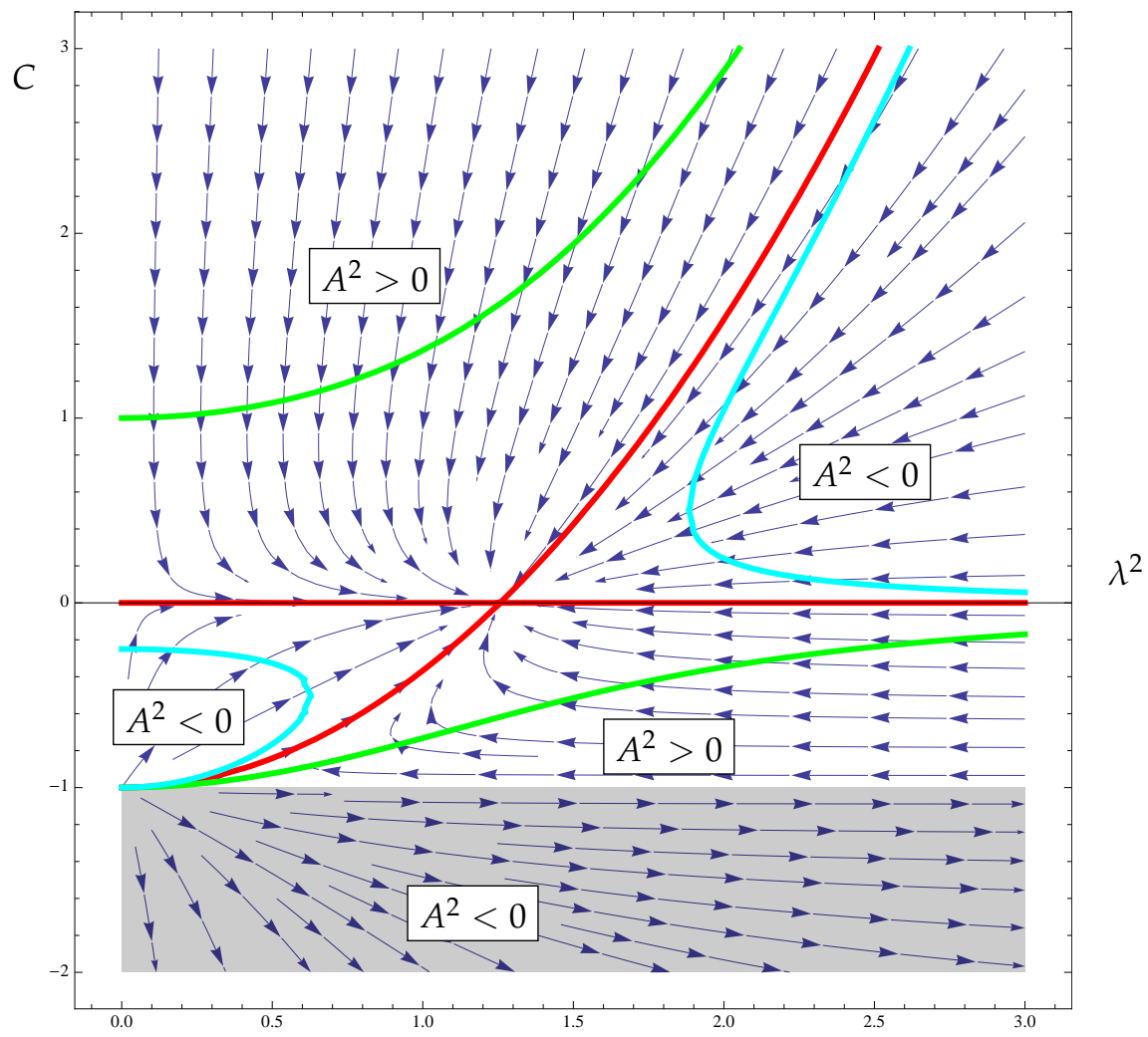

Figure 1: RG flow for $n=20$. The vertical axis shows $C$ and the horizontal one $\lambda^{2}$. The unique IR fixed point on the line $C=0$ is the undeformed SU(2) wZNW model. The red line depicts $C_{ \pm}[0]$, the green line $C_{ \pm}[1]$, the cyan line $C_{ \pm}[-i / 2]$. The flow is not defined in the grey region $C<-1$.

The $\left(\lambda^{2}, C\right)$ plane is separated into four regions by red lines corresponding to $C=C_{0}^{ \pm}(\lambda)$ (this is the locus where the flatness condition is satisfied without improvement term $I_{\mu}$ ). In these regions, $A^{2} \gtrless 0$. The green line in Fig. 4 corresponds to the locus $\left\{C_{1}^{ \pm}\right\}$(i.e. $A^{2}=1$ ), while the cyan lines represent $\left\{C_{-i / 2}^{ \pm}\right\}$(i.e. $A^{2}=-1 / 4$ ). Those are typical examples and, by varying the value of $A^{2}$, the green and cyan lines cover all of either regions.

Up to this point we have implicitly considered $A^{2}>0$ since we interpreted it as resulting from the boundary term contribution in Eq. (2.18). The RG flow analysis suggests that this condition is too strong and that it is possible to analytically continue to $A^{2}<0$. In fact, this is natural from the point of view of the $\mathrm{O}(3)$ sigma model at $C=-1$ (which is by itself the analytical continuation of our Lorentzian worldsheet) where the boundary contribution is interpreted as a Hopf (instanton) term. Moreover, the conserved charges depend only on $A^{2}$ and no imaginary term is generated by the continuation. 


\section{Conclusion and Discussion}

In this letter we have discussed the Yangian symmetry of the squashed wzNw model. The wz term is fixed by dimensionality up to an overall coefficient. For a special value of this coefficient, a flat conserved current can be constructed directly. For general values, it is possible to construct a flat conserved current using a current improvement term.

Although we have discussed only the case of the squashed sphere, it is straightforward to carry out the same analysis for warped AdS spaces via double Wick rotations. It would also be interesting to consider a generalization of the present analysis to higher-dimensional cases such as squashed $S^{7}$, possibly using arguments similar to the ones given in [34].

Another avenue of research is to include world-sheet fermions. For symmetric spaces, the relation between world-sheet fermions and non-local charges are well studied (for example, see $[4,35]$ and related work in [31,36-38]). Similarly, we expect in our case that it is possible to find a flat conserved current (and a Yangian algebra) by including appropriate world-sheet fermions. Of course, it would be even more interesting to study space-time fermions by introducing deformations of super Lie groups as target spaces.

The next natural step consists in understanding the physical implications of the Yangian symmetry that we have described. In particular it will be interesting to see whether the existence of the infinite charges implies complete integrability in the sense of Liouville. In fact, since the squashed sigma model is thought to be a continuum limit of the xxz model [39] (even though the Yangian symmetry is closely related to the xxx model), it is possible that we have found a form of "partial" integrability, related to a "subclass" of the soliton solutions in the squashed sigma model.

\section{Acknowledgments}

We would like to thank Y. Hatsuda, H. Kawai, T. Okada and M. Staudacher for illuminating discussions and S. Reffert for comments on the manuscript. This work was supported by the scientific grants from the ministry of education, science, sports, and culture of Japan (No. 22740160), and in part by the Grant-in-Aid for the Global COE Program "The Next Generation of Physics, Spun from Universality and Emergence" from the Ministry of Education, Culture, Sports, Science and Technology (MEXT) of Japan. DO acknowledges support by the World Premier International Research Center Initiative (WPI Initiative).

\section{References}

[1] M. Lüscher and K. Pohlmeyer, "Scattering Of Massless Lumps And Nonlocal Charges In The Two-Dimensional Classical Nonlinear Sigma Model," Nucl. Phys. B 137 (1978) 46.

[2] D. Bernard, “Hidden Yangians In 2-D Massive Current Algebras”, Commun. Math. Phys. 137 (1991) 191. 
[3] N. J. MacKay, "On the classical origins of Yangian symmetry in integrable field theory," Phys. Lett. B 281 (1992) 90 [Erratum-ibid. B 308 (1993) 444].

[4] E. Abdalla, M. C. Abdalla and K. Rothe, "Non-perturbative methods in two-dimensional quantum field theory," World Scientific, 1991.

[5] V. G. Drinfel'd, "Hopf Algebras and the Quantum Yang-Baxter Equation," Sov. Math. Dokl. 32 (1985) 254; "A New realization of Yangians and quantized affine algebras," Sov. Math. Dokl. 36 (1988) 212.

[6] E. Brezin, C. Itzykson, J. Zinn-Justin and J. B. Zuber, "Remarks About The Existence Of Nonlocal Charges In Two-Dimensional Models," Phys. Lett. B 82 (1979) 442.

[7] J. M. Maldacena, "The large N limit of superconformal field theories and supergravity," Adv. Theor. Math. Phys. 2 (1998) 231 [Int. J. Theor. Phys. 38 (1999) 1113] [arXiv:hep-th/9711200].

[8] G. Mandal, N. V. Suryanarayana and S. R. Wadia, "Aspects of semiclassical strings in AdS(5)," Phys. Lett. B 543 (2002) 81 [arXiv:hep-th/0206103].

[9] I. Bena, J. Polchinski and R. Roiban, "Hidden symmetries of the $\mathrm{AdS}_{5} \times S^{5}$ superstring," Phys. Rev. D 69 (2004) 046002 [arXiv:hep-th/0305116].

[10] K. Zarembo, "Strings on Semisymmetric Superspaces," arXiv:1003.0465 [hep-th].

[11] D. T. Son, "Toward an AdS/cold atoms correspondence: a geometric realization of the Schroedinger symmetry," Phys. Rev. D 78 (2008) 046003 [arXiv:0804.3972 [hep-th]].

[12] K. Balasubramanian and J. McGreevy, "Gravity duals for non-relativistic CFTs," Phys. Rev. Lett. 101 (2008) 061601 [arXiv:0804.4053 [hep-th]].

[13] S. Kachru, X. Liu and M. Mulligan, "Gravity Duals of Lifshitz-like Fixed Points," Phys. Rev. D 78 (2008) 106005 [arXiv:0808.1725 [hep-th]].

[14] S. Schafer-Nameki, M. Yamazaki and K. Yoshida, "Coset Construction for Duals of Non-relativistic CFTs," JHEP 0905 (2009) 038 [arXiv:0903.4245 [hep-th]].

[15] S. Deser, R. Jackiw and S. Templeton, "Three-Dimensional Massive Gauge Theories," Phys. Rev. Lett. 48 (1982) 975; S. Deser, R. Jackiw and S. Templeton, "Topologically massive gauge theories," Annals Phys. 140 (1982) 372 [Erratum-ibid. 185 (1988 APNYA,281,409-449.2000) 406.1988 APNYA,281,409].

[16] D. Anninos, W. Li, M. Padi, W. Song and A. Strominger, "Warped $\mathrm{AdS}_{3}$ Black Holes," JHEP 0903 (2009) 130 [arXiv:0807.3040 [hep-th]].

[17] J. M. Bardeen and G. T. Horowitz, "The extreme Kerr throat geometry: A vacuum analog of $\mathrm{AdS}_{2} \times \mathrm{S}^{2}, "$ Phys. Rev. D 60 (1999) 104030 [arXiv:hep-th/9905099].

[18] M. Guica, T. Hartman, W. Song and A. Strominger, "The Kerr/CFT Correspondence," Phys. Rev. D 80 (2009) 124008 [arXiv:0809.4266 [hep-th]].

[19] D. Israel, C. Kounnas, D. Orlando and P. M. Petropoulos, "Electric / magnetic deformations of $S^{3}$ and $\mathrm{AdS}_{3}$, and geometric cosets," Fortsch. Phys. 53 (2005) 73 [arXiv:hep-th/0405213];

[20] D. Israel, C. Kounnas, D. Orlando and P. M. Petropoulos, "Heterotic strings on homogeneous spaces," Fortsch. Phys. 53 (2005) 1030 [arXiv:hep-th/0412220].

[21] S. Detournay, D. Orlando, P. M. Petropoulos and P. Spindel, "Three-dimensional black holes from deformed anti de Sitter," JHEP 0507 (2005) 072 [arXiv:hep-th/0504231]. 
[22] D. Orlando, "String Theory: Exact solutions, marginal deformations and hyperbolic spaces," Fortsch. Phys. 55 (2007) 161-282. [hep-th/0610284].

[23] G. Compere, S. Detournay and M. Romo, "Supersymmetric Gódel and warped black holes in string theory," Phys. Rev. D 78 (2008) 104030 [arXiv:0808.1912 [hep-th]].

[24] S. Detournay, D. Israel, J. M. Lapan and M. Romo, "String Theory on Warped $\mathrm{AdS}_{3}$ and Virasoro Resonances," arXiv:1007.2781 [hep-th].

[25] D. Orlando and L. I. Uruchurtu, "Warped anti-de Sitter spaces from brane intersections in type II string theory," arXiv:1003.0712 [hep-th].

[26] E. D'Hoker and P. Kraus, "Charged Magnetic Brane Solutions in $\mathrm{AdS}_{5}$ and the fate of the third law of thermodynamics," JHEP 1003 (2010) 095 [arXiv:0911.4518 [hep-th]]; "Holographic Metamagnetism, Quantum Criticality, and Crossover Behavior," arXiv:1003.1302 [hep-th].

[27] I. Kawaguchi and K. Yoshida, "Hidden Yangian symmetry in sigma model on squashed sphere," JHEP 1011 (2010) 032 [arXiv:1008.0776 [hep-th]].

[28] D. Orlando, S. Reffert, L. I. Uruchurtu, "Classical Integrability of the Squashed Three-sphere, Warped AdS3 and Schroedinger Spacetime via T-Duality," J. Phys. A A44 (2011) 115401. [arXiv:1011.1771 [hep-th]].

[29] A. M. Polyakov, “Hidden Symmetry Of The Two-Dimensional Chiral Fields," Phys. Lett. B 72 (1977) 224.

[30] J. M. Evans, M. Hassan, N. J. MacKay and A. J. Mountain, “Local conserved charges in principal chiral models," Nucl. Phys. B 561 (1999) 385 [arXiv:hep-th/9902008].

[31] J. M. Evans, M. Hassan, N. J. MacKay and A. J. Mountain, "Conserved charges and supersymmetry in principal chiral and WZW models," Nucl. Phys. B 580 (2000) 605 [arXiv:hep-th/0001222].

[32] I. Bakas, D. Orlando, P. M. Petropoulos, "Ricci flows and expansion in axion-dilaton cosmology," JHEP 0701 (2007) 040. [hep-th/0610281].

[33] D. Orlando, "Axion-dilaton cosmology, Ricci flows and integrable structures," Nucl. Phys. Proc. Suppl. 171 (2007) 304-305. [hep-th/0702011 [HEP-TH]].

[34] M. Hatsuda and S. Tomizawa, "Coset for Hopf fibration and Squashing," Class. Quant. Grav. 26 (2009) 225007 [arXiv:0906.1025 [hep-th]]

[35] E. Abdalla and M. Forger, "Integrable non-linear $\sigma$ models with fermions," Comm. Math. Phys. 104 (1986) 123.

[36] T. L. Curtright and C. K. Zachos, "Nonlocal Currents For Supersymmetric Nonlinear Models," Phys. Rev. D 21 (1980) 411; "Supersymmetry and the nonlocal Yangian deformation symmetry," Nucl. Phys. B 402 (1993) 604 [arXiv:hep-th/9210060].

[37] L. E. Saltini and A. Zadra, "Algebra of Non-Local Charges in Supersymmetric Non-Linear Sigma Models," Int. J. Mod. Phys. A 12 (1997) 419 [arXiv:solv-int/9511007].

[38] J. M. Evans, N. J. MacKay and M. Hassan, "Conserved charges and supersymmetry in principal chiral models," arXiv:hep-th/9711140.

[39] L. D. Faddeev and N. Y. Reshetikhin, "Integrability Of The Principal Chiral Field Model In (1+1)-Dimension," Annals Phys. 167 (1986) 227. 